\title{
Rapid Adaptation Induces Persistent Biases in Population Codes for Visual Motion
}

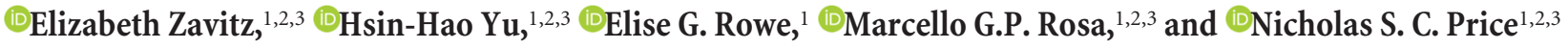 \\ ${ }^{1}$ Department of Physiology, ${ }^{2}$ Neuroscience Program, Biomedicine Discovery Institute, and ${ }^{3}$ ARC Centre of Excellence for Integrative Brain Function, \\ Monash University Node, Monash University, Clayton, VIC 3800, Australia
}

Each visual experience changes the neural response to subsequent stimuli. If the brain is unable to incorporate these encoding changes, the decoding, or perception, of subsequent stimuli is biased. Although the phenomenon of adaptation pervades the nervous system, its effects have been studied mainly in isolation, based on neuronal encoding changes induced by an isolated, prolonged stimulus. To understand how adaptation-induced biases arise and persist under continuous, naturalistic stimulation, we simultaneously recorded the responses of up to 61 neurons in the marmoset (Callithrix jacchus) middle temporal area to a sequence of directions that changed every $500 \mathrm{~ms}$. We found that direction-specific adaptation following only $0.5 \mathrm{~s}$ of stimulation strongly affected encoding for up to $2 \mathrm{~s}$ by reducing both the gain and the spike count correlations between pairs of neurons with preferred directions close to the adapting direction. In addition, smaller changes in bandwidth and preferred direction were observed in some animals. Decoding individual trials of adaptationaffected activity in simultaneously recorded neurons predicted repulsive biases that are consistent with the direction aftereffect. Surprisingly, removing spike count correlations by trial shuffling did not impact decoding performance or bias. When adaptation had the largest effect on encoding, the decoder made the most errors. This suggests that neural and perceptual repulsion is not a mechanism to enhance perceptual performance but is instead a necessary consequence of optimizing neural encoding for the identification of a wide range of stimulus properties in diverse temporal contexts.

Key words: adaptation; area MT; direction aftereffect; marmoset; middle temporal area; motion

\section{Significance Statement}

Although perception depends upon decoding the pattern of activity across a neuronal population, the encoding properties of individual neurons are unreliable: a single neuron's response to repetitions of the same stimulus is variable, and depends on both its spatial and temporal context. In this manuscript, we describe the complete cascade of adaptation-induced effects in sensory encoding and show how they predict population decoding errors consistent with perceptual biases. We measure the time course of adaptation-induced changes to the response properties of neurons in isolation, and to the correlation structure across pairs of simultaneously recorded neurons. These results provide novel insight into how and for how long adaptation affects the neural code, particularly during continuous, naturalistic vision.

\section{Introduction}

Perception is produced by the aggregate activity of populations of neurons, but linking population activity to perceptual outcomes is challenging. To approach this problem, we consider how stim-

\footnotetext{
Received Dec. 21, 2015; revised Feb. 15, 2016; accepted March 13, 2016.

Author contributions: E.Z. and N.S.C.P. designed research; E.Z., H.-H.Y., M.G.P.R., and N.S.C.P. performed research; E.Z., E.G.R., and N.S.C.P. analyzed data; E.Z. and N.S.C.P. wrote the paper.

This work was supported by National Health and Medical Research Council Project Grants APP1008287 and APP1066588, Human Frontier Science Program Career Development Award to N.S.C.P., the ARC SRl in Bionic Vision, and the ARC Centre of Excellence for Integrative Brain Function. We thank Dr. Maureen Hagan and Dr. Adam Morris for helpful comments on the manuscript. We also thank Janssen-Cilag Pty Limited for the donation of sufentanil citrate.

The authors declare no competing financial interests.

Correspondence should be addressed to Dr. Elizabeth Zavitz, 26 Innovation Walk, Monash University, Clayton, VIC 3800, Australia. E-mail: elizabeth.zavitz@monash.edu.

DOI:10.1523/JNEUROSCI.4563-15.2016

Copyright $\odot 2016$ the authors $\quad 0270-6474 / 16 / 364579-12 \$ 15.00 / 0$
}

ulus information is encoded in the responses of neurons selective for various stimulus properties. To produce perception, this pattern of activity encoded across neurons must be interpreted as a particular perceptual state, or decoded. This implies that, if the manner in which sensory information is encoded changes, the associated perceptual interpretation may be subject to errors.

Perceptual errors are particularly interesting because it is known that encoding changes often and rapidly. Following tens of seconds of exposure to a visual stimulus, single neurons change how they encode information by altering their membrane potential (Carandini and Ferster, 1997), firing rate, bandwidth, and stimulus preferences (Dragoi et al., 2000; Kohn and Movshon, 2004). As many aspects of the relationship between the stimulus and firing rate (encoding) change following adaptation, the relative strengths of the responses within a population to a test stim- 
ulus shift in an adaptor-dependent way. A prevailing theory is that the "rules" governing the decoding of this sensory activity do not change, producing systematic perceptual errors (Schwartz et al., 2007; Seriès et al., 2009); however, many aspects of how adaptation affects encoding and decoding are unknown.

Adaptation is commonly studied in the context of motion perception because sustained exposure to a moving stimulus produces vivid aftereffects. In the case of the direction aftereffect (DAE), perceptual errors are said to be repulsive: a test direction appears to be shifted away (repelled) from the adapting direction (Levinson and Sekuler, 1976). Although aftereffects represent a perceptual error, adaptation is commonly thought to be beneficial as there is evidence that it improves discrimination thresholds (Krekelberg et al., 2006; Price and Prescott, 2012), increases sensitivity (Price and Born, 2013), or makes sensory coding more efficient (Fairhall et al., 2001; Kohn, 2007; Benucci et al., 2013).

The present study focuses on the relationship between adaptation-induced changes in the activity of neurons in the middle temporal area (MT) and the DAE. The activity of MT neurons is causally linked with the perception of direction of motion (Britten et al., 1992; Salzman et al., 1992). It has been shown that adaptation to directions at or near an MT neuron's preferred direction reduces the gain and bandwidth of the tuning curve (Kohn and Movshon, 2003, 2004; Patterson et al., 2014). In addition, adaptation on the flanks of the tuning curve can shift the peak of the curve either toward the adaptor (Kohn and Movshon, 2004) or away from it, depending on stimulus configuration (Wissig and Kohn, 2012; Patterson et al., 2014). Current models combine changes in gain, preferred direction, and bandwidth across a population of neurons to produce the decoding errors that underlie the DAE (Georgeson, 2004; Kohn and Movshon, 2004; Clifford et al., 2007). It is unknown how encoding is impacted during natural vision when neurons are continuously engaged in representing a prolonged, dynamic, full-field stimulus. In a natural environment, neurons are chronically stimulated, and stimulus properties are continually changing. Are the effects of relative adaptation to the most recent stimuli sufficiently large to affect encoding, and how long do they last? Can adaptation improve perceptual performance even in the presence of decoding biases?

We examined how adaptation to individual directions within continuous stimulation affects encoding in area MT, and how these encoding changes account for decoding errors associated with the DAE. We presented a stimulus that changed direction every $500 \mathrm{~ms}$ while we recorded population activity in area MT of marmoset monkeys. Following each motion period, we observed long-lasting, direction-dependent changes in gain, bandwidth, and spike-count correlations. We decoded stimulus direction based on single-trial spiking activity and found that the model made errors consistent with the DAE. This suggests that repulsive aftereffects are a consequence of optimizing long-term identification in a stimulus space subject to adaptor-specific gain changes.

\section{Materials and Methods}

Preparation and recording. We performed extracellular recordings in three anesthetized, adult New World monkeys ( 2 male, 1 female; common marmoset, Callithrix jacchus). The experiments were conducted in accordance with the Australian Code of Practice for the Care and Use of Animals for Scientific Purposes. All procedures were approved by the Monash University Animal Ethics Experimentation Committee. Our procedures are slightly modified from the procedure described by Bourne and Rosa (2003), as described by Yu and Rosa (2014). Briefly, anesthesia was induced with alfaxalone (Alfaxan, $8 \mathrm{mg} / \mathrm{kg}$ ), and a tracheotomy and vein cannulation were performed. The animal was artificially ventilated with a gaseous mixture of nitrous oxide and oxygen (7:3). Maintenance solution with opiate anesthetic (sufentanil) and paralytic (pancuronium bromide) was infused intravenously. Atropine and phenylephrine hydrochloride eye drops were applied; then the cornea was protected, and the eyes were fitted with contact lenses to bring a monitor at $350 \mathrm{~mm}$ into focus. The ipsilateral eye was occluded. A craniotomy and durotomy were performed over area MT, and a $10 \times 10,96$ channel, "Utah" array (Blackrock Microsystems) was implanted using a pneumatic insertion tool over area MT (localized using gross anatomical landmarks, verified postmortem using receptive field maps and histology). Recordings were collected at $30 \mathrm{kHz}$ using a Cerebus system (Blackrock Microsystems). The raw voltage signal was high pass filtered at $750 \mathrm{~Hz}$, and spikes were detected based on threshold crossings.

Visual stimulus. Stimuli were presented on a VIEWPixx 3D $(1920 \times$ 1080 pixels; $520 \times 295 \mathrm{~mm}$; VPixx Technologies) positioned at a viewing distance of $350 \mathrm{~mm}$. The stimulus filled the display and consisted of a sheet of white dots on a black background. The field consisted of 0.5 dots per degree square, and each dot subtended 0.3 degrees of visual angle. Dots moved coherently with no noise in 1 of 12 directions and at either 10 degrees per second (Animal 2, Animal 3) or one of 5, 10, 20, 40, 80 degrees per second (Animal 1). In Animal 1, we have combined data across all stimulus speeds. The stimulus moved in one direction for 500 $\mathrm{ms}$ before changing to another, random, direction. Each direction was presented 600 times, for an average of 50 repeats of each consecutive pairing. To measure the tuning properties of our multiunits in the absence of adaptation, we used a similar stimulus with the same directions and appearance, but with a $500 \mathrm{~ms}$ blank (black) screen between each presentation of moving dots. This stimulus was displayed within $3 \mathrm{~h}$ of the adaptation stimulus in Animals 2 and 3, and $30 \mathrm{~h}$ before the adaptation stimulus for Animal 1. To address isolation changes, we removed channels that were minimally responsive (gain $<5$ spikes/s or circular variance (bandwidth) $>0.98$ ) in the paradigm that included blanks: 8,2 , and 1 multiunit clusters from Animals 1-3, respectively.

Assessing tuning. On every channel, the mean firing rate was calculated $50-550 \mathrm{~ms}$ after onset for each test direction regardless of adaptor, and fit with a von Mises function. If the fit had an $r^{2}$ value of at least 0.85 and was a significantly better fit than a straight-line function ( $p<0.05, F$ test $)$, the channel was included for further analysis. We included a total of 42 channels from Animal 1, 61 channels from Animal 2, and 61 channels from Animal 3 on the basis of this analysis. These fits were used to determine the relationship between the preferred directions of the units and the adaptor directions, and thus identify the adaptor closest to the antipreferred direction to act as the control condition in future analyses. Spike density functions were computed with a $30 \mathrm{~ms}$ boxcar function.

Measuring changes in responsivity. When we looked at the effects of adaptation, we used metrics that are not dependent on fits and therefore more robust to noise because the time course and number of repetitions were limited. We split the trials into $12 \times 12$ conditions, for each pairing of adapt and test direction. This resulted in 12 tuning curves, one for each adaptation condition. The control condition was the condition where the adaptor was closest to the antipreferred direction. We measured gain as the difference between the maximum and minimum firing rates (spikes per second) and expressed changes as normalized gain: gain in the test condition normalized by gain in the control condition, expressed as a percentage. We measured preferred direction from the mean vector where the magnitude associated with each direction was the firing rate. To examine the bandwidth, or sharpness of tuning, we measured circular variance. To measure spike count correlations, we calculated Pearson's $r$ for the $Z$-scored firing rates on each channel, excluding responses where $|Z|>3$.

Statistics. We used criteria of $\alpha=0.01$ and $d=0.1$ unless otherwise specified to identify statistically significant, meaningful effects. Effect sizes were measured using Cohen's d.

We used one-way ANOVAs to test for a main effect of adaptation condition on gain, preferred direction, and bandwidth; and again to test for main effect of time, or $n$-back (see Fig. 2). In those cases where a significant main effect was found, we tested each point with paired $t$ tests for means significantly different from 100 in the gain and bandwidth comparisons, and 0 in the preferred direction comparison. We used a criterion of $\alpha=0.05$ because of the relatively small number of cases in 
each condition. Benjamini-Hochberg-Yekutieli corrections for multiple comparisons were applied.

Changes in correlation strength were tested with unpaired $t$ tests comparing the distributions for the $n$-back indicated and the distribution of 9-back for each ROI (see Fig. 4). Benjamini-Hochberg-Yekutieli corrections for multiple comparisons were applied. ANOVAs were performed to test for a main effect of $n$-back on correlation change, but these were overpowered ( $>1,000,000$ degrees of freedom); so although they were statistically significant, we do not consider them meaningful. Pearson's correlations were computed on the distributions of spike-count correlation and mean firing rate. These correlations were compared using Fisher's $Z$ test.

Differences in performance over time were measured while decoding performance was at a plateau so the distributions were approximately Gaussian (see Fig. 5). Performance with shuffled trials was compared with performance with unshuffled trials with a paired $t$ test. We used an ANOVA to verify that the error biases at 1-back and 2-back showed a main effect of adaptation condition, then $t$ tests to compare the error bias in each condition to 0 . The biases at 1-back and 2-back were compared with each other using paired $t$ tests. The main effect of adaptation condition on the performance of the model was verified with a one-way ANOVA and a criterion of 0.05 . Bonferroni post hoc tests were used to determine which adaptation conditions produced significantly different performance.

Decoding model. We used a multinomial GLM to produce continuous estimates of stimulus direction from neural responses to the 12 classes of presented stimuli. Once trained, the GLM produced a probability for each of the classes, given the activity on a single test trial. The 12 probabilities were combined into a vector sum that produced a continuous estimate of stimulus direction. For each animal, we selected 20 subpopulations of 20 units from the pool of significantly direction-selective units. On a given trial, we summed the number of spikes for each unit over a 30 ms window to produce a response vector, $r=\left[r_{1}, \ldots, r_{N}\right]$, where $N$ was 20, the number of units. The probability that a given stimulus direction $\theta_{s}$ evoked the response vector $r$ was estimated with a logistic GLM (Eqs. 1, $2)$. The model weights $\left(w_{d i}\right)$ were iteratively refined until classification performance was optimized via penalized maximum likelihood. To produce a continuous guess, the probabilities for each direction were combined as a vector sum (Eq. 3) to produce a continuous prediction of stimulus direction $\left(\theta_{\text {pred }}\right)$ as follows:

$$
\begin{gathered}
p_{d}\left(\theta_{s}=\theta_{d}\right)=f\left(\sum_{i \in[1 \mathrm{~N}]} w_{d i} r_{i}\right) \\
f(a)=1 /\left(1+e^{-a}\right) \\
\theta_{\text {pred }}=\tan ^{-1}\left(\frac{\sum_{d} p_{d} \sin \theta_{d}}{\sum_{d} p_{d} \cos \theta_{d}}\right)
\end{gathered}
$$

The model was trained on $80 \%$ of randomly selected trials for supervised learning. The remaining $20 \%$ of trials were used to produce the identification predictions reported here. Each subpopulation was tested 5 times with different training and test trials.

Decoding performance. The identification performance (percentage correct) of the decoder was evaluated by coding a guess as "correct" if it was within $15^{\circ}$ of the displayed stimulus direction. To produce a time course (see Fig. $6 a$ ), the $30 \mathrm{~ms}$ integration window was offset from the stimulus onset by different amounts of time. In this case, the model was trained only on a $30 \mathrm{~ms}$ window of spiking activity $900 \mathrm{~ms}$ after adaptor onset and tested on the first $30 \mathrm{~ms}$ of neural activity after stimulus onset through to $1500 \mathrm{~ms}$ after stimulus onset in $30 \mathrm{~ms}$ increments. Variance was estimated by decoding 20 different subpopulations of 20 units each for each animal.

Biased decoders. The biased decoders were given only the trials where the test was counterclockwise or clockwise of the adaptor, respectively. They were trained on 2500 trials (as the number of counterclockwise and clockwise trials were not equal) and tested on the remaining trials. Their performance, weights, and predicted DAE were compared with an unbi- ased decoder that was also trained on 2500 trials. Fixing the number of training trials was a necessary control because training tends to increase model weights, so a larger training corpus, and thus more training, produces higher weights on average.

Predicted DAE. To measure the error distribution, we calculated the difference between the stimulus direction and the predicted direction trial-by-trial. We sorted these error measurements based on the difference between the adapting and test stimulus directions using the same $n$-back analysis protocol where every stimulus direction is both an adaptor and a test in different trials. For each difference between the test and adaptation directions, the mean of the error distribution was taken as the predicted DAE. This was measured in 20 different subpopulations of units to obtain estimates of variance.

Decorrelating trials. Pairwise correlations between units were removed by shuffling trials independently for each recorded unit. To maintain the effects of adaptation, 1-back adapt-test direction pairs were preserved and shuffled together.

\section{Results}

\section{Brief, continuous adaptation changes neuronal encoding}

Adaptation is known to change how neurons encode visual stimuli, but the precise nature of these changes is usually measured only under artificial conditions where a small variety of stimuli are displayed, the test immediately follows the adaptor, and the stimulus is restricted to a small part of the visual display (but see Priebe et al., 2002; Glasser et al., 2011). In V1, adaptation periods of less than a second induce changes in encoding (Müller et al., 1999; Dragoi et al., 2002). Equally rapid adaptation in MT allowed us to present a dynamic visual sequence and examine how encoding changed in this more complex paradigm.

We recorded multiunit activity in area MT of 3 anesthetized marmoset monkeys (Callithrix jacchus) with a 96-electrode "Utah" array (Blackrock Microsystems). We used a stimulus comprising a field of coherently moving dots, which changed direction every $500 \mathrm{~ms}$. Twelve stimulus directions were presented in random order, and each direction was repeated 600 times for a total of 7200 trials (Fig. 1a). For such a stimulus, we can treat every trial as both an adaptor (for the trials that follow) and a test (for the trials that preceded it). To reveal the relative effect of adaptation to each direction, we sorted the responses to each test direction based on the previous adapting direction, giving $12 \times 12$ adapt-test pairings.

The activity of two multiunits illustrates how the response to the direction that evokes the largest response (within $15^{\circ}$ of each unit's true preferred direction) is affected by the direction of motion in the preceding adaptation period (Fig. $1 b, d$ ). Tuning curves based on responses to the test period, but calculated separately for each adapting direction, suggest that adaptation predominantly affects response gain in these units (Fig. 1c,e). Notably, firing rates are reduced following adaptation close to the preferred direction (yellow) compared with adaptation to motion in the opposite direction (blue), and these effects are sustained throughout the test period. This shows that the effects of $500 \mathrm{~ms}$ adaptation last at least $500 \mathrm{~ms}$. Response gain was largest after adaptation to the antipreferred direction, which we refer to as the control condition. Gain reductions associated with other adaptors showed a systematic dependence on the difference between the adaptor and preferred direction.

Stimulus-dependent changes in response properties are apparent even though some degree of adaptation is always present during continuous stimulation. The responses following an antipreferred adaptor are similar to those measured following a blank stimulus (Fig. $2 a-c$ ). Using antipreferred adaptation as a reference means that at our baseline, the gain of the responses is usu- 


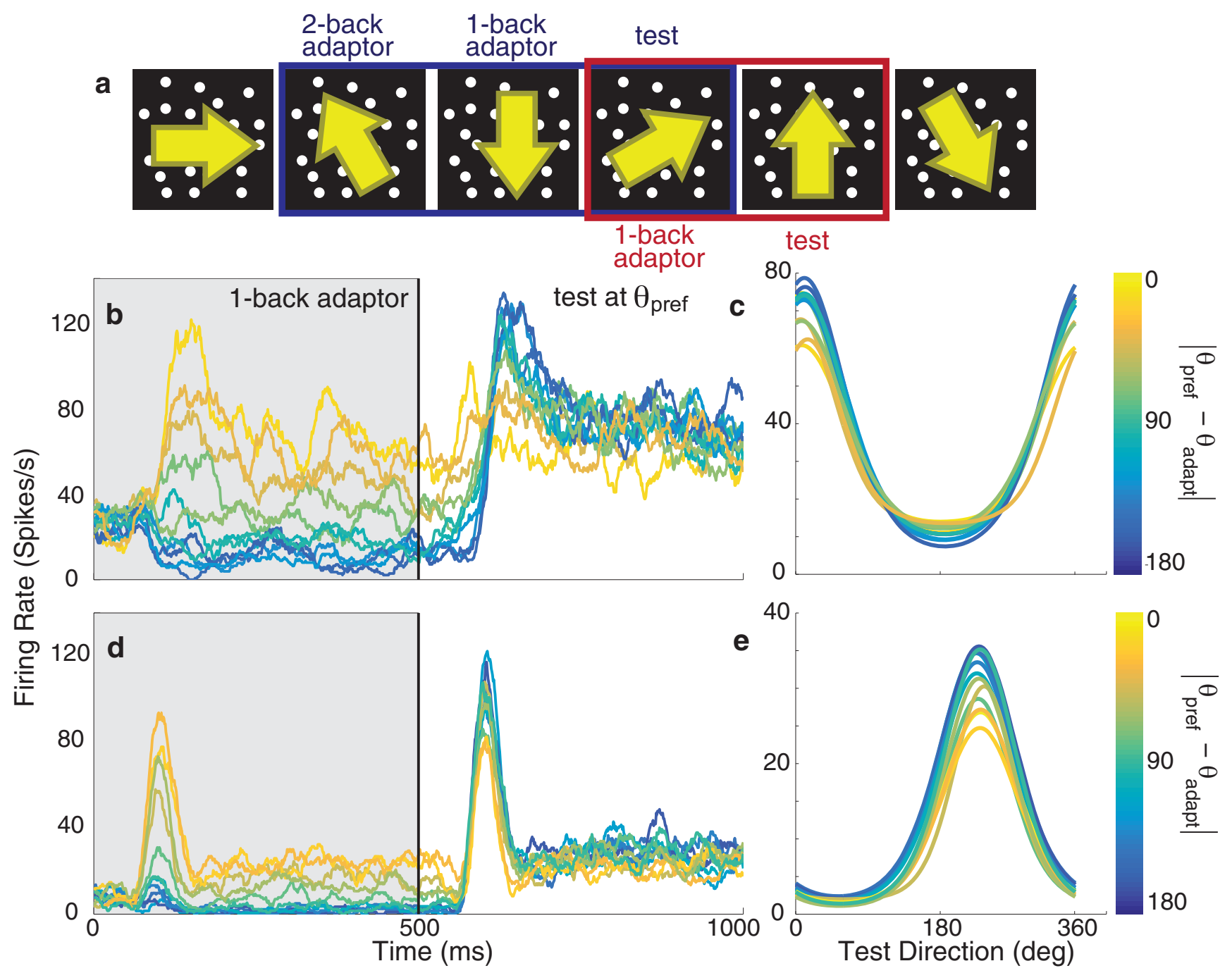

Figure 1. Firing rates evoked by a test stimulus depend on prior adaptation. $\boldsymbol{a}, \mathrm{A}$ random sequence of directions was presented. Each motion period lasted $500 \mathrm{~ms}$, and each direction was presented 600 times. A period of motion acted both as a test and an adaptor for the subsequent directions. Responses were sorted based on each adapt-test pairing (12 $\times 12$ conditions). $\boldsymbol{b}, \boldsymbol{d}$, Responses of two units to 12 adapt-test pairings in which the test was always the preferred direction. Average responses to each adaptor are shown in the shaded region ( $0-500 \mathrm{~ms})$, followed by responses to the preferred direction $(500-1000 \mathrm{~ms})$. Color represents the magnitude of the difference between the adaptor and the unit's preferred direction, where yellow is $0^{\circ}$ (the adaptor is the preferred direction) and blue is $180^{\circ}$ (the adaptor is the antipreferred direction; or control). Firing rate is affected in an adaptor-dependent way. c, $\boldsymbol{e}$, von Mises fit to postadaptation tuning curves for the units shown in $\boldsymbol{b}, \boldsymbol{d}$, averaged from responses $50-550 \mathrm{~ms}$ after onset of the test stimulus. Change in tuning curves is mainly in response gain.

ally lower (paired $t$ test, $p<0.01$ for Animals 2 and 3) (Fig. 2a) and bandwidth is narrower than in the absence of adaptation (paired $t$ test, $p<0.01$ for all animals) (Fig. $2 b$ ), as would be expected, given that the neurons are somewhat adapted already. The preferred direction of the neurons is consistent (Fig. 2c) regardless of baseline. We can attribute these differences partially to contrast adaptation: in the no-adaptation condition, a blank screen preceded the onset of the dots and motion.

On average, gain following adaptation to the preferred direction was $80 \%-90 \%$ of the gain following adaptation in the control direction (Fig. $2 d$ ). We observed a main effect of adaptation condition on gain $\left(p<0.01\right.$ for all animals; $F_{(\mathbf{A 1} 23,963)}=9.75$; $\left.F_{(\mathbf{A} 223,1408)}=2.00 ; F_{(\mathbf{A 3} 23,1402)}=6.75\right)$ and gradual decreases in the effect of adaptation as the difference between the adaptor and the preferred direction increased. Adaptors $>60^{\circ}$ from the preferred direction had no significant impact on gain relative to the control (unpaired $t$ test, $p>0.05$ ). We observed an increase in bandwidth in two of the three animals studied $\left(F_{(\mathbf{A 1} 23,963)}=3.69\right.$, $p<0.01 ; F_{(\mathbf{A} 223,1178)}=1.15, p=0.28 ; F_{(\mathbf{A} 323,1402)}=6.22, p<$
0.01) (Fig. 2e), and a main effect of adaptation on preferred direction only in Animal $1\left(F_{(\mathbf{A 1} 123,963)}=3.83, p=<0.01\right.$; $\left.F_{(\mathbf{A} 223,1178)}=1.29, p=0.16 ; F_{(\mathbf{A} 323,1402)}=0.52, p=0.97\right)$ (Fig. $2 f$ ). Our finding that changes in gain depend on distance from adaptor to preferred direction are in agreement with previous research. Others have shown direction selective reductions in gain (Kohn and Movshon, 2003; Patterson et al., 2014), and it is well established that we expect gain reductions proportional to the unit's response to the adaptor in general (Solomon and Kohn, 2014). That we did not find systematic changes in preferred direction as has been previously reported (Kohn and Movshon, 2004) may appear surprising but can be attributed to differences in our stimuli, which we elaborate on in the Discussion.

We reported the data from individual animals separately because we had sufficient statistical power in each case alone, and revealing the differences between cases accounts for an important source of variance and indicates which results should be replicable. We expect that the differences we have observed between animals can be attributed to two factors: (1) cortical neurons are 

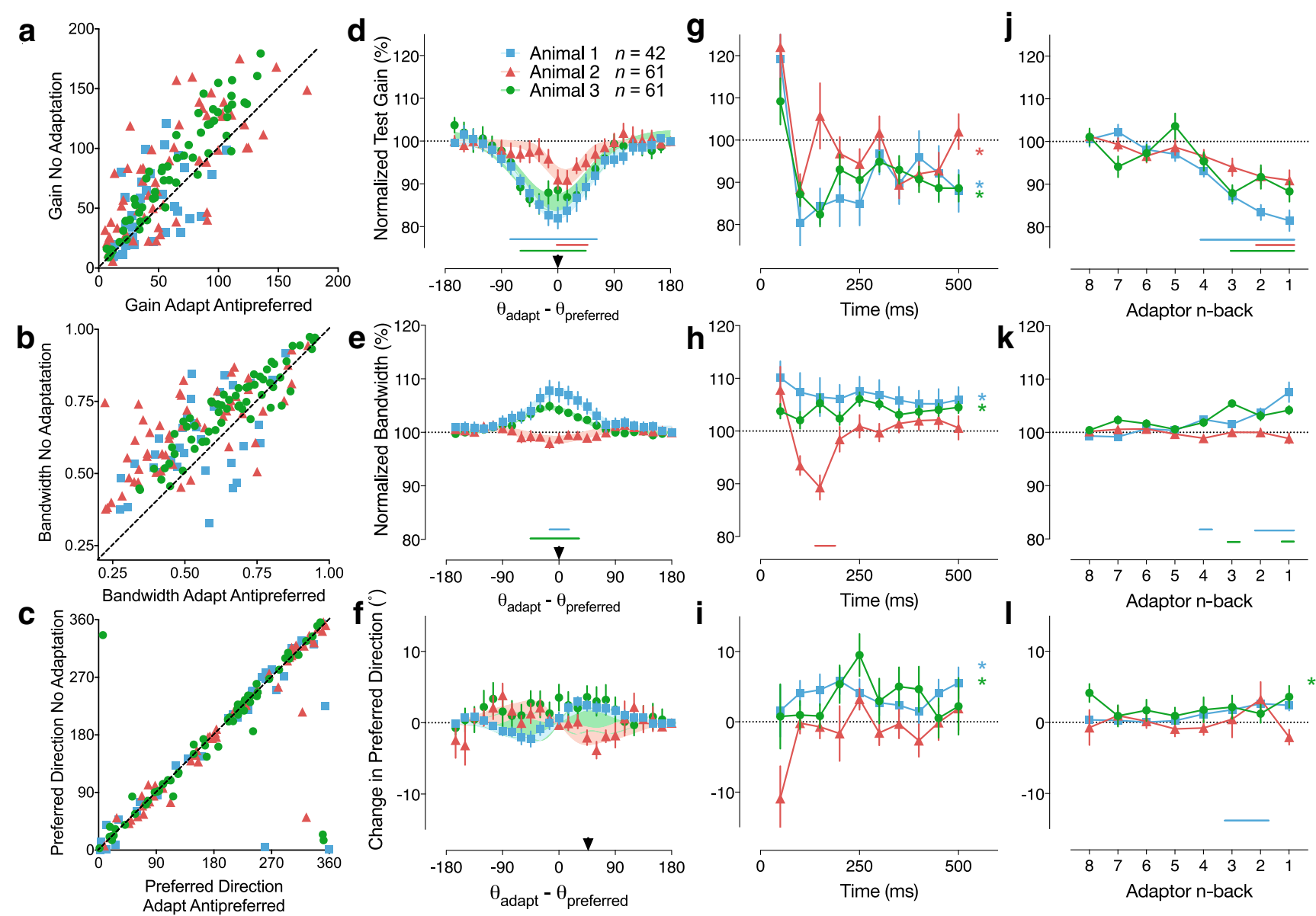

Figure 2. Direction-dependent and persistent changes in encoding following adaptation. $\boldsymbol{a}-\boldsymbol{c}$, Gain, bandwidth (circular variance), and preferred direction measured following $500 \mathrm{~ms}$ of blank screen (No Adaptation) and $500 \mathrm{~ms}$ of Antipreferred Direction Adaptation. $\boldsymbol{d}$, Changes in gain based on the angular distance between the adaptor and the preferred direction using a sliding $30^{\circ}$ bin. Filled region represents the mean and $95 \%$ confidence interval of Gaussian fits to the raw data. Gain is normalized for each unit relative to the control (antipreferred) adaptor. Horizontal lines indicate bins where the normalized gain is significantly different from $100 \%$ ( $t$ test, $p<0.05$ ). Arrows indicate the condition that is expanded in $\boldsymbol{g}$-i.e, $\boldsymbol{f}$, Same as in $\boldsymbol{d}$, for bandwidth and preferred direction. $\boldsymbol{g}$, After adaptation at the preferred direction, gain changes were evident throughout the test period. Gain changes are shown in $50 \mathrm{~ms} \mathrm{spike} \mathrm{counting} \mathrm{windows} \mathrm{throughout} \mathrm{the} \mathrm{test} \mathrm{period.} \mathrm{Horizontal}$ lines indicate significant post hoc tests. *Conditions that are significantly affected by adaptation, but not time. $\boldsymbol{h}$, Same as in $\boldsymbol{d}$ for bandwidth. The cases varied in how the bandwidth of multiunits shifted following adaptation. $\boldsymbol{i}$, Same as in $\boldsymbol{d}$ for preferred direction at following adaptation on the flank of the tuning curve $\left(45^{\circ}\right)$. $\boldsymbol{j}$, Adapt-test pairings were constructed for 1-back to 8 -back adapt-test pairs. $\boldsymbol{d}, \boldsymbol{g}$, The 1-back condition; a consecutive adapt-test pairing. Following preferred direction adaptation, gain was reduced for up to 4 motion periods ( $2 \mathrm{~s}$ ). Horizontal lines indicate statistically significant gain reduction. Error bars indicate SE. $\boldsymbol{k}, \boldsymbol{I}$, Same as in $\boldsymbol{j}$ for bandwidth and preferred direction.

diverse, and the limited number of neurons we acquired from each case represent subtly different samples; and (2) the state of the brain varied between recording sessions. In particular, as we discuss in detail later, Animal 3 experienced strong fluctuations in global activity levels throughout the experiment.

\section{Gain reductions outlast intervening stimulation}

Adaptation is a time-sensitive process; and given the brief adaptation period, we expected that the effects on encoding would also be short-lived (Dragoi et al., 2002). Previously, we integrated each unit's activity over the entire $500 \mathrm{~ms}$ response to the test stimulus, but we thought it likely that the effects of adaptation would be strongest in the earlier part of that period. To look at the time course of adaptation within the test period, we sampled spiking activity at $50 \mathrm{~ms}$ intervals. The reduction in gain is established over the first $100 \mathrm{~ms}$ of stimulus onset, but does not change significantly between 100 and $550 \mathrm{~ms}\left(F_{(\mathbf{A 1} 18,369)}=0.98, p=0.45\right.$; $\left.F_{(\mathbf{A} 28,540)}=1.93, p=0.05 ; F_{(\mathbf{A 3} 8,540)}=1.522, p=0.15\right)($ Fig. $2 g)$. Gain from 100 to $550 \mathrm{~ms}$ is significantly lower than $100 \%$ ( $t$ test, $p<0.01)$. We only observed an effect of time on bandwidth in Animal $2\left(F_{(\mathbf{A 1} 8,369)}=0.09, p=0.99 ; F_{(\mathbf{A} 28,540)}=3.79, p<0.001\right.$;
$\left.F_{(\mathbf{A 3} 8,540)}=1.09, p=0.37\right)$ (Fig. 2h). Bandwidth for Animals 1 and 3 was significantly broadened ( $t$ test, $p<0.01)$, and briefly, but significantly narrowed for Animal 2 (post hoc $t$ test, $p<0.01$ ). Adaptation affects the preferred direction of neurons when it occurs on a flank, not the preferred direction. We used the flanking direction of $45^{\circ}$ and observed no effect of time on the change in preferred direction $\left(F_{(\mathbf{A} 18,369)}=0.5, p=0.82 ; F_{(\mathbf{A} 28,540)}=\right.$ $\left.0.51, p=0.85 ; F_{(\mathbf{A 3} 38540)}=1.00, p=0.44\right)$ (Fig. 2i). Preferred directions were significantly shifted in Animals 1 and 3 ( $t$ test, $p<$ 0.01 ). Based on the analysis for a $45^{\circ}$ adaptor (Fig. $2 f$, arrow, $i, l$ ), Animal 3 appears to exhibit an attractive shift in preferred direction, but this is not a systematic effect: the shift is repulsive for the flanking adaptor direction $-45^{\circ}$. Overall, for all cases, we observe very little fluctuation in the impact of adaptation over the course of the test period.

In natural vision, adaptation can occur because of a bias in the stimulus statistics over time (i.e., the "adaptor" is the orientation or direction that occurs more frequently than any others) (Benucci et al., 2013; Dhruv and Carandini, 2014). Adaptation also occurs after one-off exposure to a brief stimulus, as shown above. Here we examine the persistence of encoding changes in the face 

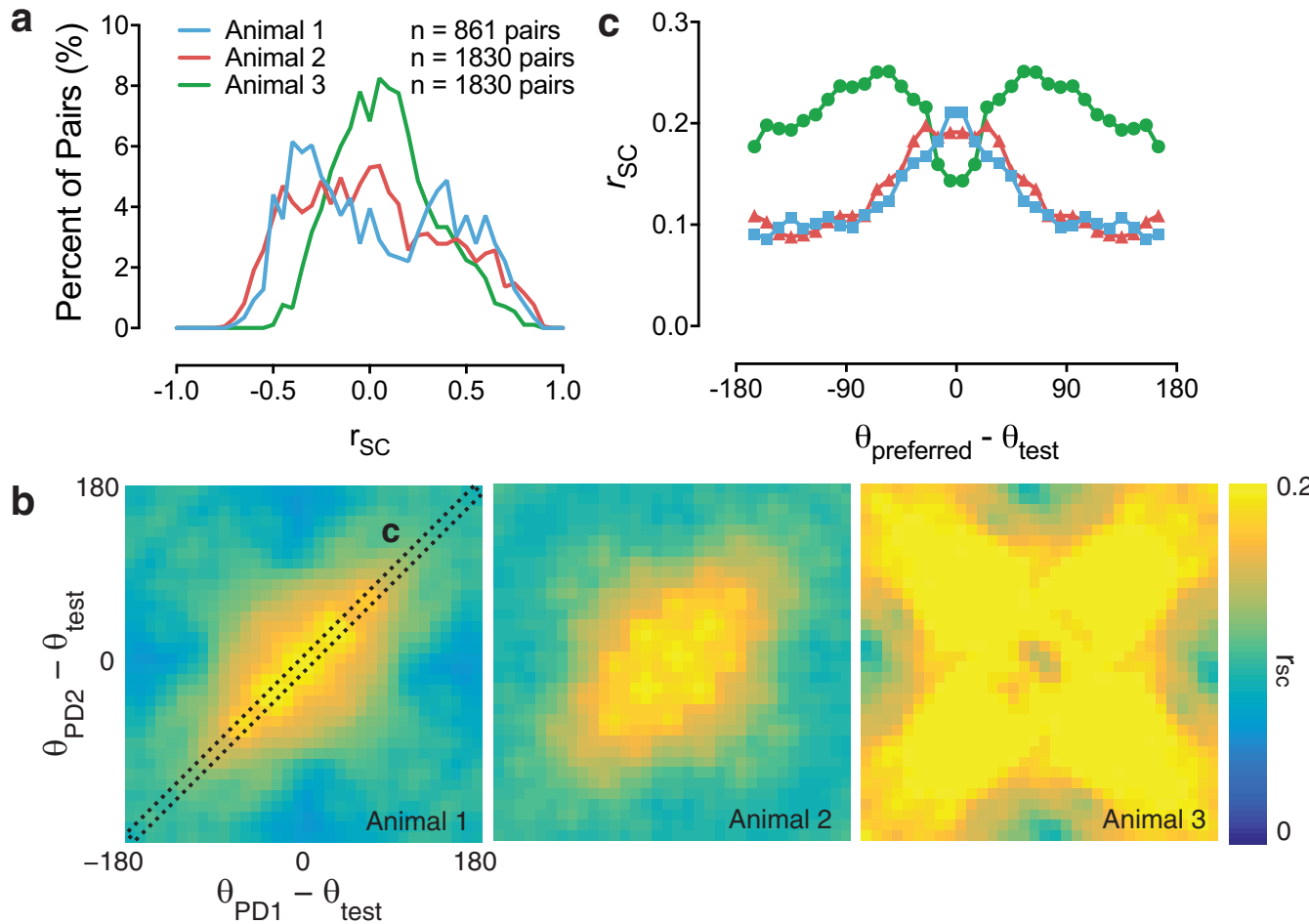

Figure 3. Stimulus dependence of spike count correlations between pairs of neurons. $a$, Histograms of correlations measured between pairs of recorded multiunits calculated for firing rates in all trials. Correlations measured in Animals 1 and 2 are similarly distributed; correlations measured in Animal 3 are higher and follow a narrower distribution. $\boldsymbol{b}$, Correlations grouped according to the difference between the preferred directions of the neurons and the test direction for all 3 animals. Animals 1 and 2 show a strong relationship between correlation strength and the direction of the stimulus when the stimulus is near the preferred directions of neurons with similar preferences (dashed diagonal box). Data have been symmetrized and smoothed using a Gaussian kernel with SD $30^{\circ}$. , Data from the diagonal indicated in $\boldsymbol{b}$, with different cases superimposed. Animal 3 differs from Animals 1 and 2 in both the magnitude and stimulus sensitivity of the measured correlations.

of intervening stimulation. To do this, we used an $n$-back adaptor analysis paradigm (Fischer and Whitney, 2014). Instead of sorting trials by the direction immediately preceding the test direction (1-back), we sorted them by the direction 2-8 stimulus periods earlier (Fig. $2 j-l$ ). Across trials, this averages the effect of all directions in intervening motion periods and reveals the direction-specific effects of an $n$-prior stimulus. These effects are measured on top of the chronic contrast adaptation, and a baseline level of some degree of direction-specific "top-up" in the intervening stimulus periods. In all animals, we found significant adaptor-specific changes in gain over time $\left(F_{(\mathbf{A 1} 7,328)}=19.06\right.$, $\left.p<0.01 ; F_{(\mathbf{A} 27,480)}=4.33, p<0.01 ; F_{(\mathbf{A} 37,480)}=5.849, p<0.01\right)$, and post hoc $t$ tests determined that these changes were significant $(p<0.05)$ up to three intervening adaptation periods (4-back, or $2 \mathrm{~s}$ ) (Fig. 2j). We also observed significant changes in bandwidth over time for Animals 1 and $3\left(F_{(\mathbf{A 1} 7,328)}=9.74, p<0.01\right.$; $\left.F_{(\mathbf{A 2} 7,480)}=1.19, p=0.31 ; F_{(\mathbf{A 3} 7,480)}=6.42, p<0.01\right)$, these were also significant in post hoc tests for up to $2 \mathrm{~s}$ (Fig. $2 k$ ). The effect of adaptation on preferred direction only changed over a longer time course for Animal $1\left(F_{(\mathbf{A 1} 7,328)}=3.54, p<0.01 ; F_{(\mathbf{A 2} 7,480)}=\right.$ $\left.0.79, p=0.60 ; F_{(\mathbf{A} 37,480)}=0.67, p=0.37\right)$. Post hoc tests showed these changes lasted from 1 to 2 intervening adaptors (Fig. $2 l$ ). This shift in preferred direction $\left(2.4^{\circ}-2.6^{\circ}\right)$ is much smaller than the $9.3^{\circ}$ previously reported (Kohn and Movshon, 2004).

\section{Adaptation decorrelates population activity}

Adaptation is known to affect network-level properties in addition to single-unit encoding (Gutnisky and Dragoi, 2008; Benucci et al., 2013). To examine the effect of adaptation on the population, we measured spike-count correlations $\left(r_{\mathrm{sc}}\right)$ for each pair of tuned multiunits in each animal $(N=861, N=$
$1830, N=1830$ ). For each trial, we integrated the number of spikes $>500 \mathrm{~ms}$ and then $z$-scored across all trials for each multiunit.

To examine the degree of shared variability across the population regardless of stimulus direction, we first measured the correlations observed over all trials (Fig. $3 a$ ). The distributions were very similar for Animals 1 and 2, whereas the correlations measured for Animal 3 were higher and followed a narrower distribution. To evaluate how these correlations depended on the stimulus, we measured them under each of the 12 stimulus test directions and then sorted them according to the difference between each unit's preferred direction $\left(\theta_{\mathrm{PD} 1}\right.$ and $\left.\theta_{\mathrm{PD} 2}\right)$ and the test direction $\left(\theta_{\text {test }}\right)$ (Fig. $3 b$ ). In Animals 1 and 2, we found that the correlations were highest when both neurons preferred a direction similar to the stimulus direction, whereas Animal 3 had abnormally high correlations across most stimulation conditions. In the case where the neurons have similar preferred directions (Fig. 3c), Animals 1 and 2 clearly show the highest correlations when the test direction is at the preferred direction, in agreement with previous findings (Solomon et al., 2014). Animal 3 follows an opposite trend. During the experiment, we observed strong fluctuations in global activity in Animal 3. It appears as though these fluctuations imposed a correlation structure that impairs our ability to look at how spike count correlations depend on the stimulus. For this reason, we omitted Animal 3 from our analyses on the effects of adaptation on correlations. The range of these correlations $(0.1-0.2)$ and the mean values for each animal $\left(\mu_{\mathrm{A} 1}=0.06 ; \mu_{\mathrm{A} 2}=0.04 ; \mu_{\mathrm{A} 3}=0.11\right)$ are consistent with previous reports of correlations in MT of awake macaques between 0.1 (Huang and Lisberger, 2009) and 0.13 (Cohen and Newsome, 2008). 

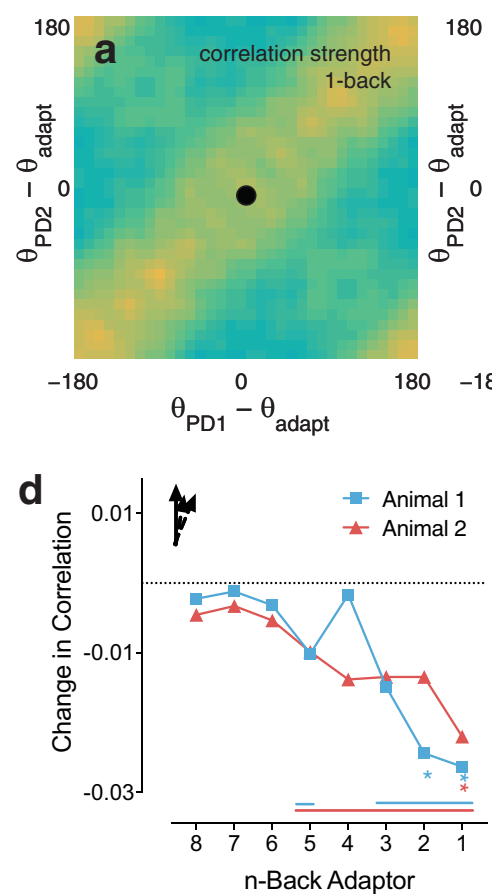
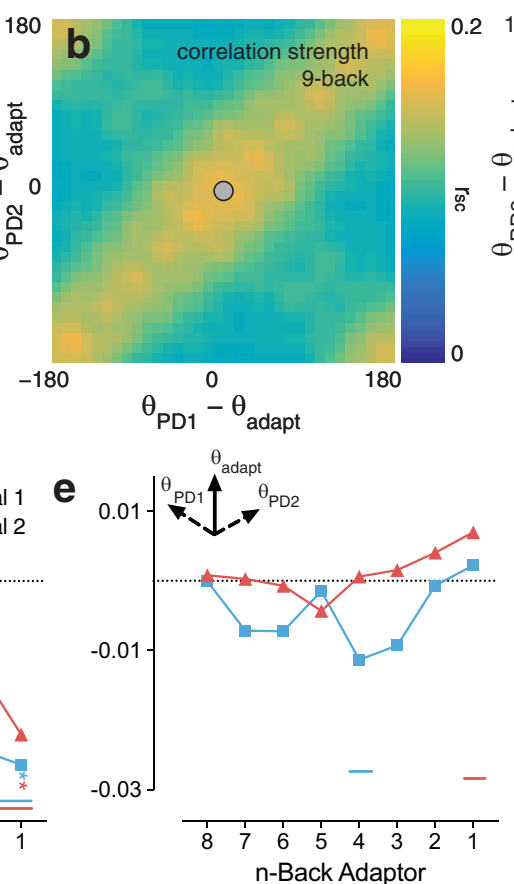
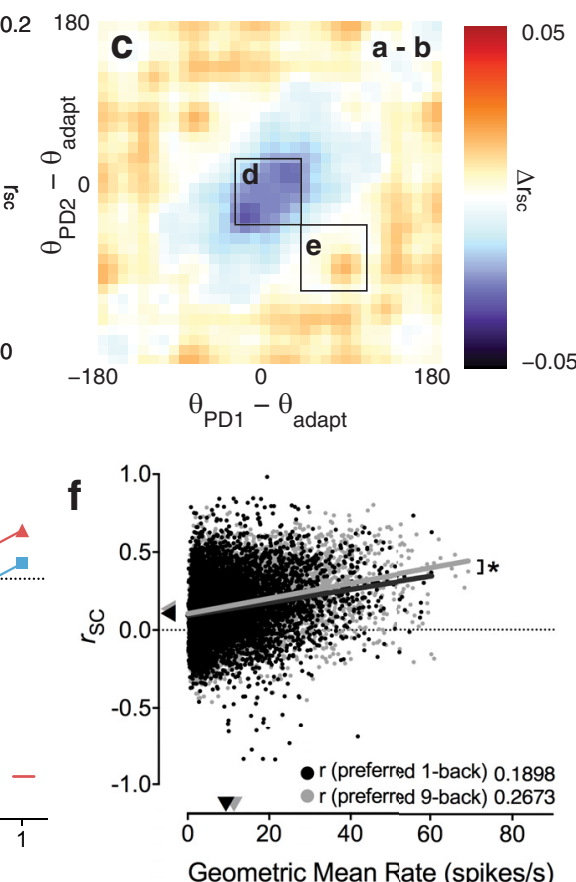

Figure 4. Adaptation affects correlation structure in a direction-dependent way. $\boldsymbol{a}$, Spike-count correlations ( $\left.r_{s c}\right)$ measured during the test period but grouped according to the relationship between the adapting direction and the preferred directions of the two units. The preferred directions of the two multiunits are noted as $\theta_{\mathrm{PD} 1}$ and $\theta_{\mathrm{PD} 2}$. Yellow diagonal running from bottom left to top right indicates that, when units prefer similar directions, they have higher correlations. The center of the diagonal, marked with a black circle, shows where two units with similar preferred directions are adapted with a stimulus near their preferred directions. $\boldsymbol{b}$, A baseline correlation structure, analyzed in an identical manner to $\boldsymbol{a}$, but taken from the adaptor 9 -back from the test stimulus. $\boldsymbol{c}$, Change in correlation structure immediately following adaptation calculated by subtracting $\boldsymbol{b}$ from $\boldsymbol{a}$. Correlations are reduced when units with similar preferred directions have been strongly adapted. Correlations are also increased when the pair of neurons prefer directions that are $>60^{\circ}$ away from the adaptor and $120^{\circ}-150^{\circ}$ away from each other (off the main diagonal). $\boldsymbol{d}$, ROI analysis of correlation changes for each animal. The mean correlation change for each animal in the central Rol illustrated in c is shown for each animal, from 1-back to 8-back. Schematic represents an example of a relationship between the adaptor (solid arrow) and the preferred directions of the two units (dashed arrows). For both animals, correlations are reduced for several seconds following adaptation. Horizontal lines indicate statistical significance (unpaired $t$ test, $p<0.01$ ). *Significant results with effect sizes $>0.1$. $\boldsymbol{e}$, Same as in $\boldsymbol{d}$ for the off-center R0I shown in c. Correlations are sometimes significantly changed, but the effect size is $<0.1$. $f$, Correlation between firing rate and spike count correlation under adaptation (black, condition shown with black point in $\boldsymbol{a}$ ) and at baseline (gray, gray point in $\boldsymbol{b}$ ). Spike count correlation strength is correlated with firing rate in both cases, but this dependence is significantly reduced under adaptation, suggesting that the reduction in correlations after adaptation is not a simple consequence of the observed reduction in gain.

We combined the data from Animals 1 and 2 for illustrative purposes and sorted correlations measured during the test period based on adapting rather than test direction. This demonstrates that neurons with similar preferred directions have the strongest correlations, but also highlights that these correlations are reduced after adaption to a near-preferred direction, as there are stronger correlations in the top and bottom corners than in the center of the diagonal (Fig. 4a). To quantify changes in correlations following adaption, we took the correlation structure after 9-back adaptation as a baseline (Fig. $4 b$ ), as direction-specific effects of adaptation on gain had dissipated well before this time (Fig. $2 j$ ), but the precise choice of the time window for the baseline did not qualitatively affect the results.

To reveal the adaptor-specific change in correlation structure, we subtracted the baseline correlation structure from the 1-back correlation structure. Adaptation decreased correlations when the members of the pair of units had similar (within $30^{\circ}$ ) preferred directions and had been adapted near that direction (Fig. $4 c$, inset, $d$ ). They also appeared to increase when the units had disparate preferred directions (separated by $120^{\circ}-150^{\circ}$ ) that were both $60^{\circ}-120^{\circ}$ away from the adaptor (Fig. $4 c$, inset, $e$ ).

Within the ROIs indicated in Figure $4 c$, we aggregated the data in $\pm 30^{\circ}$ bins. When both units preferred a direction similar to the adapting direction (Fig. 4d), Animals 1 and 2 showed a statistically significant decrease in correlations from 1-back through 3-back ( $t$ test, $p<0.01$ ). The size of the effect (Cohen's $d$ ) was
$>0.1$ for up to 2-back $(1 \mathrm{~s})$. The increase in correlations for mismatched preferred directions and adaptor (Fig. $4 e$ ) is statistically significant $(t$ test, $p<0.01)$ at some time points, but the effect size (Cohen's $d$ ) is $<0.1$ in each case. ANOVAs were conducted to explore a main effect of $n$-back on the center and flanking ROIs. Both ANOVAs were statistically significant; but given the number of correlations measured, the tests are overpowered and the results not informative.

The magnitude of spike count correlations is known to increase with spiking rate (de la Rocha et al., 2007); so to determine whether the reductions in correlations we observed are a natural consequence of the observed changes in gain, we measured the correlation between $r_{\mathrm{sc}}$ and the geometric mean of the firing rates of the pair of neurons in both the 1-back and 9-back conditions (Fig. $4 f$ ). Here we focus only on conditions in which both neurons were adapted near their preferred direction (Fig. $4 a, b$, black and gray circles). Not only are $r_{\mathrm{sc}}$ and mean firing rate reduced following 1-back adaptation (Fig. $4 f$, arrows), but importantly the strength of the correlation between them is significantly reduced relative to 9-back adaptation (Fisher's $Z, Z=-5.3$ ). This is evidence that the reduction in spike count correlations is not a simple consequence of reductions in gain.

These results illustrate that adaptation changes the structure of neuronal correlations, mainly by suppressing correlations between those neurons with the same preferred directions following an adaptor close to their preferred directions. Furthermore, 
these changes in correlation happen rapidly alongside changes in gain. This suggests that changes in spike count correlations following adaptation are not due to slow processes, such as network reorganization.

\section{Changes in encoding following rapid adaptation produce a prediction error consistent with the DAE}

Although rarely encountered in natural vision, prolonged exposure to a constant stimulus can lead to profound changes in perception. For example, after viewing motion to the right for many seconds, vertical motion appears to be moving slightly to the left. This phenomenon is referred to as the DAE (Levinson and Sekuler, 1976; Clifford et al., 2007; Wenderoth and Wiese, 2008). We have demonstrated that our stimulus paradigm induces adaptordependent changes in neural responses, but it remains to be seen whether the decreases in gain we observed on a short timescale are sufficient to bias decoding outcomes in a way that emulates the DAE.

To test this, we trained a multinomial GLM (Friedman et al., 2010; Berens et al., 2012; Qian et al., 2013) to classify each test direction. As input, the decoder took spike counts from 20 simultaneously recorded multiunits over a $30 \mathrm{~ms}$ time window. To cross-validate, we trained the decoder on $80 \%$ of all trials and tested on the remaining $20 \%$ of trials to estimate performance. We also trained the model at only one time point and tested at all the others. We resampled our populations of neurons, running analyses on 20 different, randomly selected, subpopulations of 20 units from each animal with 5 different sets of training and test trials of each direction, for a total of 100 decoding simulations per time point per animal. The model output the probabilities that each of the 12 directions had produced the observed activity. The 12 outputs were combined into a continuous response using a vector sum that took the classifier probability as the magnitude for its respective direction. A trial was considered "correct" if the estimate was within $15^{\circ}$ of the stimulus direction.

Throughout a sequence of three stimuli, decoding performance is well above chance ( $40 \%-60 \%$ correct vs $8.3 \%$ chance) (Fig. 5a). These results were produced when decoders were trained $900 \mathrm{~ms}$ after adaptor onset (Fig. $5 a$, arrow), but decoding performance depends only weakly on time. The only exception is that decoding performance drops briefly after each direction onset $(0,500$, and $1500 \mathrm{~ms})$ because of the latency of neural responses (i.e., although the stimulus has changed, the population is still representing the previous direction). The decoder generalizes well from the adaptor to the test. This is not surprising because every trial is both a test and an adaptor so they are b
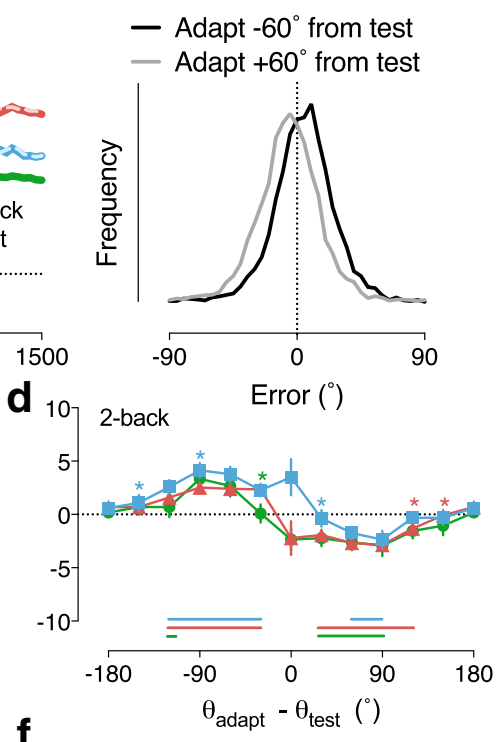

f

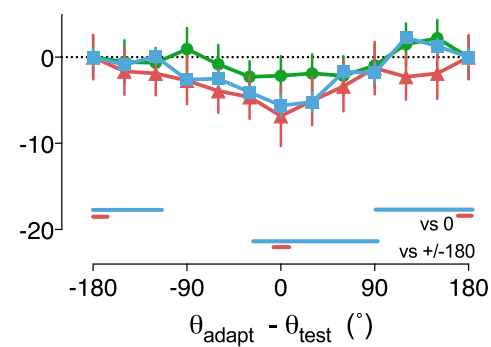

Figure 5. Decoding stimulus direction from population activity predicts the DAE. $\boldsymbol{a}$, Decoding performance over time. The decoder is trained on $30 \mathrm{~ms}$ of spiking activity from a subpopulation of 20 units measured $400 \mathrm{~ms}$ after test onset and tested on a extremely well; decoding performance is the same for all stimuli displayed in $1500 \mathrm{~ms}$ (adaptor, 1-back test, 2-back test) (one-way ANOVA, $p>0.05)$. The brief period of poor performance following a stimulus change $(t=0,500,1000)$ reflects the neuronal e latency. Removing the pairwise correlations (dashed lines) has no substantial effect on absolute performance. Shaded area represents the response to the adaptor. Arrow indicates the time point at which the model was trained. $\boldsymbol{\nabla}, 1$-back and 2-back

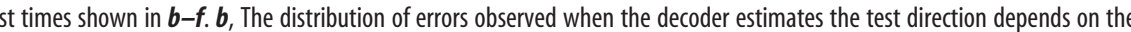
列 f the test (gray) has a negative shifted distribution. c, Mean error bias for all adapt-test pairs during the 1-back test period (tested (1) We observe a repulsive DAE: predicted test directions are shifted away from the direction of the adapting stimulus. Shuffling the trials (dashed lines) did not affect the error bias. *Bias for the correlated and uncorrelated data was significantly different. Lines indicate conditions for which the error was significantly different from $0(p<0.01)$ for each , Same as in c, for the 2-back test period (tested at 1300 ms after adaptor onset; Fig. 1a, $\mathbf{\nabla}$ ). The error bias is reduced with an intervening stimulus period. Error bars indicate SEM. * Conditions where the 2-back bias was significantly different from the 1-back aftereffect. $\boldsymbol{e}, \boldsymbol{f}$, The overall decoding performance depends on the difference between the adapting and test directions, but are most similar, and highest when they are most different. Horizontal lines indicate the results of a subset of the Bonferronicorrected post hoc tests. The remainder of the post hoc tests are reported in Table 1.

statistically identical overall. Moreover, although the decoder is trained to identify all directions ( $80 \%$ of total direction onsets) at a single time point, it generalizes to time points in a subsequent motion period as well as to different time points within the same period.

To assess the role of spike count correlations $\left(\mathrm{r}_{\mathrm{sc}}\right)$ in decoding, we removed them by randomly shuffling the trials of each adaptor test pairing across repetitions, independently for each recording channel, before training the decoding model. We found that trial-by-trial correlations between pairs of units had a statistically significant impact on performance $(p<0.01)$, but the effect of removing these correlations was very small $(\mathrm{d}<0.1$; maximum/ mean difference A1: 2.5\%/0.1\%; A2: 1.9\%/-0.2\%) (Fig. 5a, 
dashed lines). Neural decoding is often strongly impaired by the presence of correlations, so this finding is unusual. We expect it is because most examinations of the role correlations play in coding use two-class linear discriminant decoding, which linearly divides classes in the space of firing rates. Spike count correlations interact with the discriminant and improve or impair decoding if they are parallel or perpendicular to the discrimination boundary, respectively (Averbeck et al., 2006; Moreno-Bote et al., 2014). In a multiclass problem, as we used here, the space is separated repeatedly, once for each class. With multiple discriminants, the relationship between the correlations and the task will be less straightforward. We measured the prediction error on every test trial while noting the difference between the adapting direction and the test direction. Ultimately, we had 12 distributions of errors: one for each difference between adapting and test direction. Histograms for two of these distributions $\left(-60^{\circ}\right.$ and $\left.60^{\circ}\right)$ are pictured in Figure $5 b$. To measure the predicted DAE, we took the circular mean of the error distribution.

In the model, as in human observers, the direction and magnitude of the error distribution offset depended on the difference between the adapting and test stimuli (Fig. $5 c$ ). The aftereffect peaked when the adaptor was within $60^{\circ}-90^{\circ}$ of the test, producing average repulsive shifts of $4^{\circ}-6^{\circ}$ in direction estimate: tests clockwise of the adaptor appeared more clockwise, and vice versa for counterclockwise pairings. The main effect of adaptor relative to test direction was statistically significant $(p<0.01)$ for both 1-back $\left(F_{(\mathbf{A 1} 12,247)}=44.54 ; F_{(\mathbf{A 2} 12,247)}=77.28 ; F_{(\mathbf{A} 3112,247)}=\right.$ $32.93)$ and 2-back $\left(F_{(\mathbf{A} 112,247)}=24.54 ; F_{(\mathbf{A} 212,247)}=35.20\right.$; $\left.F_{(\mathbf{A} 312,247)}=15.52\right)$ adaptors. Those conditions with a bias significantly different from zero (post hoc $t$ tests, $p<0.01$ ) are indicated with horizontal lines. We found that the error bias did not depend on trial-by-trial correlations. The decoder trained on shuffled trials (dashed lines) exhibited the same aftereffect. This predicts a novel perceptual DAE that survives intervening stimulation.

As outlined above, the prediction error is strongly influenced by the difference between the test and adapting directions, but this bias appears to be largely independent of absolute decoding performance. We measured the deviation from average performance along with the bias (Fig. $5 e, f$ ), and found that, when performance was affected by adaptation, it was lowest when the test and adaptor were similar directions, and highest when the test and adaptor were farthest apart. This trend was statistically significant in two out of three animals in the 1-back $\left(F_{(\mathbf{A 1} 12,247)}=\right.$ $11.24, p<0.01 ; F_{(\mathbf{A} 212,247)}=1.48, p=0.13 ; F_{(\mathbf{A} 312,247)}=3.05$, $p<0.01)$ and 2-back $\left(F_{(\mathbf{A 1} 12,247)}=6.85, p<0.01 ; F_{(\mathbf{A 2} 12,247)}=\right.$ $\left.2.21, p<0.05 ; F_{(\mathbf{A} 312,247)}=1.73, p=0.06\right)$ conditions. Notably, any difference in overall decoding performance did not follow the effect of bias. Post hoc tests $(p<0.05)$ showed that those trials where the adaptor and test were similar are significantly worse than those where they were opposite. All significant pairwise comparisons are reported in Table 1.

It is important to note that, despite the variability between animals we observed in changes in encoding in Figures 3 and 4, we observe a very consistent effect of adaptation on prediction bias in our decoding analysis. This suggests that the most consistent changes in encoding, such as gain change, are sufficient to produce a DAE.

These results demonstrate that shifts in encoding that occur following brief stimulation in a continuous paradigm can shift the population representation to produce biases in decoding and adaptation-dependent decoding errors, but persistent biases are not the primary cause of decoding errors.
Table 1. The effect of adaptation condition on decoding performance ${ }^{a}$

\begin{tabular}{|c|c|c|c|c|c|}
\hline \multicolumn{2}{|l|}{ Animal 1} & \multicolumn{2}{|l|}{ Animal 2} & \multicolumn{2}{|l|}{ Animal 3} \\
\hline 1-back & 2-back & 1-back & 2-back & 1-back & 2-back \\
\hline-180 vs -30 & -180 vs 0 & - & -180 vs 0 & -150 vs 0 & - \\
\hline-180 vs 0 & -180 vs 30 & $\begin{array}{l}\text { No significant } \\
\text { main effect }\end{array}$ & 180 vs 0 & -150 vs 30 & $\begin{array}{l}\text { No significant } \\
\text { main effect }\end{array}$ \\
\hline-180 vs 60 & -150 vs 0 & - & - & 90 vs 0 & - \\
\hline-180 vs 90 & -120 vs 0 & - & - & 120 vs 0 & - \\
\hline-150 vs 0 & -120 vs 30 & - & - & - & - \\
\hline-120 vs 0 & 120 vs -90 & - & - & - & - \\
\hline-90 vs 0 & 120 vs -60 & - & - & - & - \\
\hline-60 vs 0 & 120 vs -30 & - & - & - & - \\
\hline-30 vs 0 & 120 vs 0 & - & - & - & - \\
\hline-30 vs 90 & 120 vs 30 & - & - & - & - \\
\hline 90 vs 0 & 150 vs -30 & - & - & - & - \\
\hline 120 vs 0 & 150 vs 0 & - & - & - & - \\
\hline 150 vs -30 & 150 vs 30 & - & - & - & - \\
\hline 150 vs 0 & 180 vs 0 & - & - & - & - \\
\hline 150 vs 30 & 180 vs 30 & - & - & - & - \\
\hline 150 vs 60 & & - & - & - & - \\
\hline 180 vs -30 & & - & - & - & - \\
\hline 180 vs 0 & & - & - & - & - \\
\hline 180 vs 30 & & - & - & - & - \\
\hline 180 vs 60 & & - & - & - & - \\
\hline 180 vs 90 & & - & - & - & - \\
\hline
\end{tabular}

${ }^{a}$ The adaptor direction pairs listed here are those for which post hoc tests indicate significantly different decoding performances $(p<0.05)$.

\section{The DAE is a consequence of compromises arising from a balanced stimulus set}

When units are ordered by their preferred direction, they form a population response curve that reliably identifies direction despite noise in individual population members (Paradiso, 1988; Pouget et al., 2000). It is often presumed that adaptor-dependent gain reductions produce repulsive aftereffects because by reducing the gain of neurons with nearby preferred directions, they push the peak of the population curve away (Jin et al., 2005; Schwartz et al., 2007; Seriès et al., 2009). We observed that the population response curve (Fig. $6 a$ ) had a Gaussian profile with units whose preferred direction matches the stimulus having the highest responses. As the above theoretical work has suggested, we also observed that the response curve was shifted counterclockwise of average on those trials when the test was clockwise of the adaptor $\left(\theta_{\text {adapt }}-\theta_{\text {test }}>0\right.$; Fig. $6 b$, dark lines $)$, and vice versa on trials when the test was counterclockwise $\left(\theta_{\text {adapt }}-\theta_{\text {test }}<0\right.$; Fig. $6 b$, light lines).

The multinomial regression we used learned a linear model for each of the 12 classes (stimulus directions), so each decoder produced $12 \times 20$ weights: a weight for each unit for each class. We measured the average weight for each unit across the 20 subpopulations and arranged these weights based on the difference between the class and the preferred direction of the unit (Fig. $6 c$ ). We found that the weights the model learned for each class produced a template of the expected population activity for that class.

The population response is shifted clockwise or counterclockwise depending on the relationship between the test and the adaptor (Fig. 6b). These conflicting shifts may produce a weighting that describes neither shift optimally when averaged across all possible trials. To remove this conflict, we constructed two biased sets of stimuli: clockwise pairings $\left(\theta_{\text {adapt }}-\theta_{\text {test }}>0\right)$ only, and counterclockwise $\left(\theta_{\text {adapt }}-\theta_{\text {test }}<0\right)$ adapt-test pairings only. We trained decoders on the biased pairings that produced the differences in Figure $6 b$ and tested them at the 1-back time point (900 ms). We found that, when the model was trained on a subset 
of only counterclockwise or only clockwise trials, the model's templates shifted to follow the population activity (Fig. $6 d$ ) and, not surprisingly, the size of the DAE was reduced.

These results serve to support longheld intuition about how adaptation in populations of neurons produces repulsive aftereffects: population activity following adaptation is distorted by direction-selective changes in neural responses to resemble activity to stimuli more different from the adaptor (Sutherland, 1961; Coltheart, 1971; Wainwright, 1999; Schwartz et al., 2007). Later stages of processing are unaware that this shift has occurred, and so the percept is repelled away from the adaptor (Seriès et al., 2009). Beyond this, we have demonstrated that perceptual repulsion is a direct consequence of optimizing a multiclass identification problem in a balanced stimulus space.

\section{Discussion}

We examined how a continuous-adaptation protocol affected the ability of populations of neurons in MT to represent motion direction. Decoding this population activity predicts systematic biases consistent with the perceptual DAE observed in humans. Previous attempts to account for the DAE through simulations have been poorly constrained, leading to conflicting suggestions that the DAE is attributable to some combination of neuronal gain changes, attractive shifts in tuning curves, and broadening bandwidth (Georgeson, 2004; Kohn and Movshon, 2004; Clifford et al., 2007; Price and Prescott, 2012), although repulsive shifts in tuning curves are observed under many adaptation conditions (Wissig and Kohn, 2012). By simultaneously recording from dozens of neurons, and using a decoding approach to relate the neuronal activity to perception, we have determined how multiunit encoding and correlations between pairs of multiunits might affect perception. Ultimately, we have demonstrated that adaptation-induced shifts in neuronal direction tuning, bandwidth, and correlations occur in some circumstances but that only neuronal gain changes are necessary to account for the perceptual DAE.

\section{Implications for neural coding}

Our findings support the common wisdom that perception is produced by a stable read-out process that is blind to adaptation in lower areas (Schwartz et al., 2007; Seriès et al., 2009). Although it is clear that population-dependent effects on neural gain occur within a few hundred milliseconds, it is unclear how much experience is necessary for the brain to correct for them so that the aftereffect is no longer apparent. Our observation that decoding errors are a result of necessary compromises in read-out over a uniform stimulus space suggests a novel way to approach this question. We hypothesize that prolonged viewing of a biased stimulus space (i.e., where many directions are clockwise of the previous direction) could reduce the magnitude of perceptual aftereffects in human observers. The amount of time, and whether feedback is required, for observers to correct their DAE could provide valuable information on the flexibility of the perceptual readout.

We showed that gain changes sufficient to produce a DAE occur throughout the neural population within the first few hundred milliseconds of motion onset. This suggests that the system is adjusting to maintain homeostasis rapidly (Benucci et al., 2013), at the cost of biasing the activity and the percept it produces. Adaptation in insects occurs on multiple timescales (Wark et al., 2009), although it is unknown how these results extend to vertebrates. If it is possible to change the read-out to account for the effect of adaptation, we would expect this change to occur more slowly. Encoding rules evolve fluidly over time as the stimulus changes, whereas decoding rules must depend on accumulating information associated with the history of both stimulation and the encoding rules. Furthermore, as long as repulsive aftereffects in any direction are equally likely, decoding rules may rarely be under pressure to change.

\section{Where does the adaptation originate?}

In this study, we have taken care to isolate the effects of directionspecific adaptation as much as possible. Previous work has sought to study adaptation by comparing exposure to a mean luminance no-adaptation condition with exposure to a moving stimulus, which induces contrast and direction adaptation; or by comparing the effects of static and moving adaptors (Petersen et al., 1985). We have displayed a continuous sequence of motion for the hour-long recording so that global activity across MT is constant, and used a control condition (adaptation in the antipreferred direction) that only differs from the test conditions in the direction of motion. As we show by comparing responses 
with and without an intervening blank period, relative changes in neuronal gain are still robustly observed during continuous stimulation, and even in the absence of contrast adaptation.

Moving gratings strongly stimulate V1 neurons, and this activation propagates to MT. Moving dots, on the other hand, evoke relatively little activation, and thus adaptation, in V1 compared with MT (Snowden et al., 1992). MT neurons are thought to change their direction tuning by attractively shifting toward the adaptor when a subset of their inputs from V1 are heavily adapted, as by a narrowband grating stimulus (Kohn and Movshon, 2004). Thus, that we did not observe systematic shifts in the preferred directions of our neurons (Fig. $2 f$ ) is not unexpected, as we used a broadband dot stimulus. We can also infer that the V1 neurons providing input to MT were only weakly adapted by the motion of the dots. Therefore, we expect that our results reflect direction-selective adaptation produced in MT neurons with very little inherited from V1.

\section{Comparison with other models}

Bayesian inference approaches to understanding adaptation, and the aftereffects it produces have been popular (Stocker and Simoncelli, 2005; Clifford et al., 2007; Schwartz et al., 2007) and successful in theoretically explaining repulsive aftereffects and improved discriminability near the adaptor. They suggest that adaptation changes the likelihood function relating neuronal responses to particular stimuli by improving the signal-to-noise ratio near the adaptor. This is typically proposed to be achieved by changing the operating range of the gain (Stocker and Simoncelli, 2005) but could also occur if adaptation decreased intertrial variability in another way. Our results are inconsistent with this theory. Although we observed a shift in error bias that depended on the relationship between the test and the adaptor (the DAE), we found no evidence that classification performance was improved following adaptation (Fig. 5e). Instead, it appears that, when adaptation has the strongest effect on the population responding to the test (i.e., when the test and adaptor are the same or similar), performance is lowest. Bayesian approaches assert that the response gain, and thus variability, of individual (Poisson) neural responses to a given test direction is reduced following preferred-direction adaptation. Yet, in a naturalistic situation with diverse adapting stimuli, response variability across adaptors at a given test direction is high. In particular, a neuron's response to a given stimulus is much lower than its mean response to that stimulus when it is most heavily adapted, which impairs the ability of the decoder to identify test directions at or near the adapting direction.

Our decoding model can be conceptualized as a series of "read-out" neurons that perform a weighted sum of inputs received from MT neurons with diverse direction tuning. The weights associated with each read-out neuron form a template, and the similarity between this template and the stimulus-evoked activity in MT represents the likelihood of the stimulus. This interpretation is similar to that of models that pool across population activity to produce a likelihood function across stimulus categories (Jazayeri and Movshon, 2006). Typically, likelihood models rely on providing the model with "labeled lines," or assumptions about the tuning of the neurons in the population. We achieve this through the period of supervised learning: the tuning properties of each neuron are inferred to be the learned optimal weights, rather than provided as an assumption of the model.

\section{Role of global correlations}

Although pairwise correlations between neurons are thought to be important in population coding (Averbeck et al., 2006; Moreno-Bote et al., 2014), we found that they did not have a meaningful impact on our classification performance or on the DAE. This is likely because the role of spike count correlations in encoding is task-dependent. In two-alternative classification problems, correlations improve decoding if they are parallel to the discriminant in the population response space and impair decoding if they are perpendicular (Averbeck et al., 2006; Moreno-Bote et al., 2014). In a 12-alternative classification task, as we have used, there are multiple discriminants so the structure of the correlations is likely to matter less. It is worth noting that generalized linear decoding models, as used here, are very forgiving of either additive or multiplicative fluctuations in global activity (Moreno-Bote et al., 2014; Lin et al., 2015), as the linear relationships in activity between channels are maintained.

\section{Role of adaptation in visual processing}

The most pronounced and consistent effect of adaptation is a reduction in firing rate. This reduction may play an important role in maintaining a low firing rate across the population over time, emphasizing sparse coding, or maintaining homeostasis (Benucci et al., 2013). Firing rate reductions affect encoding, but not decoding, and thus produce perceptual effects. These effects, from the occasionally reported improvements in discrimination to frequently observed biases in classification as demonstrated here, may be epiphenomenal. The "decoding catastrophe" represented by dynamic encoding may simply reflect that the brain prioritizes an efficient code over an accurate one.

\section{References}

Averbeck BB, Latham PE, Pouget A (2006) Neural correlations, population coding and computation. Nat Rev Neurosci 7:358-366. CrossRef Medline

Benucci A, Saleem AB, Carandini M (2013) Adaptation maintains population homeostasis in primary visual cortex. Nat Neurosci 16:724-729. CrossRef Medline

Berens P, Ecker AS, Cotton RJ, Ma WJ, Bethge M, Tolias AS (2012) A fast and simple population code for orientation in primate V1. J Neurosci 32:10618-10626. CrossRef Medline

Bourne JA, Rosa MGP (2003) Preparation for the in vivo recording of neuronal responses in the visual cortex of anaesthetised marmosets (Callithrix jacchus). Brain Res Brain Res Protoc 11:168-177. CrossRef Medline

Britten KH, Shadlen MN, Newsome WT, Movshon JA (1992) The analysis of visual motion: a comparison of neuronal and psychophysical performance. J Neurosci 12:4745-4765. Medline

Carandini M, Ferster D (1997) A tonic hyperpolarization underlying contrast adaptation in cat visual cortex. Science 276:949-952. CrossRef Medline

Clifford CW, Webster MA, Stanley GB, Stocker AA, Kohn A, Sharpee TO, Schwartz O (2007) Visual adaptation: neural, psychological and computational aspects. Vision Res 47:3125-3131. CrossRef Medline

Cohen MR, Newsome WT (2008) Context-dependent changes in functional circuitry in visual area MT. Neuron 60:162-173. CrossRef Medline

Coltheart M (1971) Visual feature-analyzers and aftereffects of tilt and curvature. Psychol Rev 78:114-121. CrossRef Medline

de la Rocha J, Doiron B, Shea-Brown E, Josiæ K, Reyes A (2007) Correlation between neural spike trains increases with firing rate. Nature 448: 802-806. CrossRef Medline

Dhruv NT, Carandini M (2014) Cascaded effects of spatial adaptation in the early visual system. Neuron 81:529-535. CrossRef Medline

Dragoi V, Sharma J, Sur M (2000) Adaptation-induced plasticity of orientation tuning in adult visual cortex. Neuron 28:287-298. CrossRef Medline

Dragoi V, Sharma J, Miller EK, Sur M (2002) Dynamics of neuronal sensitivity in visual cortex and local feature discrimination. Nat Neurosci 5:883-891. CrossRef Medline 
Fairhall AL, Lewen GD, Bialek W, de Ruyter van Steveninck RR (2001) Efficiency and ambiguity in an adaptive neural code. Nature 412:787-792. CrossRef Medline

Fischer J, Whitney D (2014) Serial dependence in visual perception. Nat Neurosci 17:738-743. CrossRef Medline

Friedman J, Hastie T, Tibshirani R (2010) Regularization paths for generalized linear models via coordinate descent. J Stat Softw 33:1-22. Medline

Georgeson M (2004) Visual aftereffects: cortical neurons change their tune. Curr Biol 14:R751-R753. CrossRef Medline

Glasser DM, Tsui JM, Pack CC, Tadin D (2011) Perceptual and neural consequences of rapid motion adaptation. Proc Natl Acad Sci U S A 108: E1080-E1088. CrossRef Medline

Gutnisky DA, Dragoi V (2008) Adaptive coding of visual information in neural populations. Nature 452:220-224. CrossRef Medline

Huang X, Lisberger SG (2009) Noise correlations in cortical area MT and their potential impact on trial-by-trial variation in the direction and speed of smooth-pursuit eye movements. J Neurophysiol 101:3012-3030. CrossRef Medline

Jazayeri M, Movshon JA (2006) Optimal representation of sensory information by neural populations. Nat Neurosci 9:690-696. CrossRef Medline

Jin DZ, Dragoi V, Sur M, Seung HS (2005) Tilt aftereffect and adaptationinduced changes in orientation tuning in visual cortex. J Neurophysiol 94:4038-4050. CrossRef Medline

Kohn A (2007) Visual adaptation: physiology, mechanisms, and functional benefits. J Neurophysiol 97:3155-3164. CrossRef Medline

Kohn A, Movshon JA (2003) Neuronal adaptation to visual motion in area MT of the macaque. Neuron 39:681-691. CrossRef Medline

Kohn A, Movshon JA (2004) Adaptation changes the direction tuning of macaque MT neurons. Nat Neurosci 7:764-772. CrossRef Medline

Krekelberg B, van Wezel RJ, Albright TD (2006) Adaptation in macaque MT reduces perceived speed and improves speed discrimination. J Neurophysiol 95:255-270. CrossRef Medline

Levinson E, Sekuler R (1976) Adaptation alters perceived direction of motion. Vision Res 16:779-781. CrossRef Medline

Lin IC, Okun M, Carandini M, Harris KD (2015) The nature of shared cortical variability. Neuron 87:644-656. CrossRef Medline

Moreno-Bote R, Beck J, Kanitscheider I, Pitkow X, Latham P, Pouget A (2014) Information-limiting correlations. Nat Neurosci 17:1410-1417. CrossRef Medline

Müller JR, Metha AB, Krauskopf J, Lennie P (1999) Rapid adaptation in visual cortex to the structure of images. Science 285:1405-1408. CrossRef Medline

Paradiso MA (1988) A theory for the use of visual orientation information which exploits the columnar structure of striate cortex. Biol Cybern 58: 35-49. CrossRef Medline

Patterson CA, Duijnhouwer J, Wissig SC, Krekelberg B, Kohn A (2014) Similar adaptation effects in primary visual cortex and area MT of the macaque monkey under matched stimulus conditions. J Neurophysiol 111: 1203-1213. CrossRef Medline
Petersen SE, Baker JF, Allman JM (1985) Direction-specific adaptation in area MT of the owl monkey. Brain Res 346:146-150. CrossRef Medline

Pouget A, Dayan P, Zemel R (2000) Information processing with population codes. Nat Rev Neurosci 1:125-132. CrossRef Medline

Price NS, Born RT (2013) Adaptation to speed in macaque middle temporal and medial superior temporal areas. J Neurosci 33:4359-4368. CrossRef Medline

Price NS, Prescott DL (2012) Adaptation to direction statistics modulates perceptual discrimination. J Vis 12:32. CrossRef Medline

Priebe NJ, Churchland MM, Lisberger SG (2002) Constraints on the source of short-term motion adaptation in macaque area MT: I. The role of input and intrinsic mechanisms. J Neurophysiol 88:354-369. CrossRef Medline

Qian J, Hastie T, Friedman J, Tibshirani R, Simon N (2013) Glmnet for MATLLAB. http://www.stanford.edu/ hastie/glmnet_matlab/.

Salzman CD, Murasugi CM, Britten KH, Newsome WT (1992) Microstimulation in visual area MT: effects on direction discrimination performance. J Neurosci 12:2331-2355. Medline

Schwartz O, Hsu A, Dayan P (2007) Space and time in visual context. Nat Rev Neurosci 8:522-535. CrossRef Medline

Seriès P, Stocker AA, Simoncelli EP (2009) Is the homunculus "aware" of sensory adaptation? Neural Comput 21:3271-3304. CrossRef Medline

Snowden RJ, Treue S, Andersen RA (1992) The response of neurons in areas $\mathrm{V} 1$ and MT of the alert rhesus monkey to moving random dot patterns. Exp Brain Res 88:389-400. CrossRef Medline

Solomon SG, Kohn A (2014) Moving sensory adaptation beyond suppressive effects in single neurons. Curr Biol 24:R1012-R1022. CrossRef Medline

Solomon SS, Chen SC, Morley JW, Solomon SG (2015) Local and global correlations between neurons in the middle temporal area of primate visual cortex. Cereb Cortex 25:3182-3196. CrossRef Medline

Stocker AA, Simoncelli EP (2005) Sensory adaptation within a Bayesian framework for perception, pp 1291-1298. Cambridge, MA: Massachusetts Institute of Technology.

Sutherland NS (1961) Figural after-effects and apparent size. Q J Exp Psychol 13:222-228. CrossRef

Wainwright MJ (1999) Visual adaptation as optimal information transmission. Vision Res 39:3960-3974. CrossRef Medline

Wark B, Fairhall A, Rieke F (2009) Timescales of inference in visual adaptation. Neuron 61:750-761. CrossRef Medline

Wenderoth P, Wiese M (2008) Retinotopic encoding of the direction aftereffect. Vision Res 48:1949-1954. CrossRef Medline

Wissig SC, Kohn A (2012) The influence of surround suppression on adaptation effects in primary visual cortex. J Neurophysiol 107:3370-3384. CrossRef Medline

Yu H-H, Rosa MGP (2014) Uniformity and diversity of response properties of neurons in the primary visual cortex: selectivity for orientation, direction of motion, and stimulus size from center to far periphery. Vis Neurosci 31:85-98. CrossRef Medline 